\title{
LIDAR CONCEPT OF “GUANLAN" MISSION FOR SPACE OCEANOGRAPHY
}

\author{
Songhua $\mathrm{Wu}^{1,2}$ *, Weibiao Chen ${ }^{3}$ Junwu Tang ${ }^{2}$, Chaofang Zhao ${ }^{1,2}$, Ge Chen ${ }^{1,2}$, \\ ${ }^{1}$ Ocean Remote Sensing Institute, College of Information Science and Engineering, Ocean University of \\ China, Qingdao 266100, China \\ ${ }^{2}$ Laboratory for Regional Oceanography and Numerical Modeling, Pilot National Laboratory for Marine \\ Science and Technology (Qingdao), Qingdao 266237, China \\ ${ }^{3}$ Shanghai Institute of Optics and Fine Mechanics, Shanghai 201800, China \\ *Email:wush@ouc.edu.cn
}

\begin{abstract}
Among the various challenges in spaceborne radar observation of the ocean, the following two issues are probably of a higher priority: inadequate dynamic resolution, and ineffective vertical penetration. Two highly anticipated breakthroughs in the coming decade are likely to be associated with radar interferometry and ocean lidar technology, which are expected to make a substantial contribution to a sub-mesoscaleresolving and depth-resolving observation of the ocean. The planned "Guanlan" science mission comprises a dual-frequency $(\mathrm{Ku}$ and $\mathrm{Ka})$ interferometric altimetry (IA) and a near-nadir pointing ocean lidar (OL). The spaceborne active OL will ensure a deeper penetration depth and an all-time detection which leads to a layered characterization of the optical properties of the subsurface ocean. The simultaneous functioning of the $\mathrm{OL}$ and a dual-frequency $(\mathrm{Ku}$ and $\mathrm{Ka}$ ) interferometric altimetry system will allow an enhanced understanding of contributions of the atmosphere and the air-sea interface which in turn considerably reduce the error budgets of the two sensors. The OL payload is expected to partially reveal the marine food chain and ecosystem with $10-\mathrm{m}$ vertical interval in the euphotic layer, moving a significant step down to the oceanic mixed layer both dynamically and bio-optically.
\end{abstract}

\section{INTRODUCTION}

Since the advent of ocean radar satellite in 1970s ${ }^{[1]}$, its development has followed three generic trends: (i) better sampling, (ii) higher accuracy, and (iii) more variables. Better sampling of the ocean normally refers to a broader coverage, a deeper penetration, a higher spatiotemporal resolution, and a longer time series. Upgraded sensors with refined algorithms (both theoretical and empirical) are major approaches to improve the accuracy of remotely sensed parameters. Meanwhile, tremendous efforts have been devoted to testing new instrument (e.g., ocean lidar) and deriving new variables from spaceborne measurements. Ideally, the ultimate goal of ocean remote sensing is towards a quantitative derivation of all required variables with a satisfactory accuracy and a perfect sampling in near-real time. In reality, however, this can never be fully realized, but can always be gradually approached. In this paper, the concept design of the ocean lidar (OL) for Chinese Guanlan science mission is described.

As a pioneer of spaceborne lidar, the US space agency NASA initiated the Lidar In-Space Technology Experiment (LITE) in $1988^{[2]}$. Following a successful feasibility study with an aerosol and cloud lidar onboard the Space Shuttle of Discovery in 1994, NASA and the French space agency CNES launched their joint mission of CALIPSO (Cloud Aerosol Lidar and Infrared Pathfinder Satellite Observation) in $2006{ }^{[3]}$, which transformed the satellite lidar technology from experimental to operational. In the years that followed, a dozen of spaceborne lidars have been proposed by several national and international space agencies such as NASA and ESA (European Space Agency).

The CALIPSO mission has provided valuable byproducts for oceanographic studies during the past decade (see Hostetler et al. ${ }^{[4]}$ for a recent review). Behrenfeld et al. ${ }^{[5]}$ use CALIOP measurements to quantify global ocean phytoplankton biomass and total particulate organic carbon stocks. Lu et al. ${ }^{[6]}$ find significant relationships between integrated subsurface backscatter and chlorophyll-a concentration, as well as particulate organic carbon, which indicate a potential use of CALIPSO lidar to estimate global chlorophyll-a 
and particulate organic carbon concentrations. $\mathrm{Lu}$ et al. (2016) introduce an approach for estimating the ocean subsurface layer-integrated backscatter and particulate backscattering coefficient from CALIOP $30^{\circ}$ off-nadir lidar measurements. Behrenfeld et al. [7] report a decade of uninterrupted polar phytoplankton biomass cycles, and find that polar phytoplankton dynamics are categorized by "boom-bust" cycles resulting from slight imbalances in plankton predator-prey equilibria.

Compared to historical passive optical remote sensing of the ocean, spaceborne lidars have two unique advantages: First, vertical penetration into the mixed layer through which profiles of optical and even oceanographic properties can be quantitatively obtained; Second, all-time measurement independent of solar radiation can be carried out. But they also have a critical limitation that all measurements are vertically integrated without depth information. A dedicated spaceborne lidar such as the one proposed for the Guanlan science mission is therefore an urgent need for a better understanding of the truly threedimensional (3-D, rather than 2.5-D) structures in the subsurface ocean.

\section{THE OCEAN LIDAR SYSTEM}

\subsection{The lidar system}

As an active optical detection instrument capable of providing time-resolving and spectral-resolving measurements, the Guanlan ocean lidar is built to perform all-time profiling of the attenuated coefficients, the backscatter coefficients and the particle linear depolarization ratios from the sea surface to the mixed layer. Additionally, the OL is also designed to make possible measurements of the ocean fluorescence and the sea level elevation. The specifications of the OL is listed in Tab 1.

Tab 1. The specifications of the OL

\begin{tabular}{|l|l|}
\hline Parameters & Specification \\
\hline & $1064.4 \pm 0.1 \mathrm{~nm}$ \\
Wavelength & $532.2 \pm 0.05 \mathrm{~nm}$ \\
& $486.13 \pm 0.05 \mathrm{~nm}$ \\
\hline & $\geqq 0.2 \mathrm{~J} @ 1064.4 \mathrm{~nm}$ \\
Single pulse energy & $\geqq 0.15 \mathrm{~J} @ 532.2 \mathrm{~nm}$ \\
& $\geqq 0.08 \mathrm{~J} @ 486.1 \mathrm{~nm}$ \\
\hline Beam divergence & $\leq 0.2 \mathrm{mrad}$ \\
\hline Pulse width & $\sim 10 \mathrm{~ns}$ \\
\hline
\end{tabular}

\begin{tabular}{|l|l|}
\hline Telescope diameter & $1.2 \mathrm{~m}$ \\
\hline FOV & $\sim 0.3 \mathrm{mrad}$ \\
\hline $\begin{array}{l}\text { Receiver optical } \\
\text { bandwidth }\end{array}$ & $\leq 0.2 \mathrm{~nm}$ \\
\hline Height resolution & $\leq 10 \mathrm{~cm}$ \\
\hline Depth resolution & $\leq 5 \mathrm{~m}$ \\
\hline $\begin{array}{l}\text { Penetration depth of ocean } \\
\text { water }\end{array}$ & $150 \mathrm{~m} \sim 300 \mathrm{~m}$ (low orbit) \\
& $130 \mathrm{~m} \sim 240 \mathrm{~m}$ (high orbit) \\
\hline
\end{tabular}

The Guanlan OL has two fully redundant lasers, each with a narrow-linewidth laser that transmits simultaneous, co-aligned multi-wavelength (including the blue and green bands) pulses (Fig. 2). The total output pulse energy is at joule level. The two lasers are expected to cover the full mission lifetime. The primary laser is scheduled to be fired since the launch of Guanlan, while the second one serves as a backup. For the receiver, a Cassegrain telescope with lightweight silicon carbide is designed to collect backscatter signals. The diameter of the telescope is approximately 1.2 $\mathrm{m}$. For accurate measurements of the troposphere and subsurface ocean, a data acquisition subsystem with a high sensitivity and a large dynamic range is required. Therefore, an analog mode along with a photon counting mode will be operating simultaneously. To ensure data integrity and processing flexibility, high-speed waveform acquisition and variable digital discrimination techniques are adopted. The heat generated by lasers is dissipated via a radiant cooling panel. The OL is installed to the satellite platform in the overall mounting framework.

The technical route of the laser system is a solidstate main oscillation power amplifier combined with a nonlinear frequency conversion technology. Multistage laser amplifiers are used to amplify the seed laser pulses from a 1064-nm main oscillator. The frequency of the amplified high energy 1064$\mathrm{nm}$ laser is doubled to obtain 532.2-nm green laser pulses. A $355 \mathrm{~nm}$ ultraviolet laser is generated by a frequency sum of the 532.2-nm laser and the remaining 1064-nm laser. The 486.1$\mathrm{nm}$ blue laser pulses will be generated by the 355 $\mathrm{nm}$ laser pumped optical parametric oscillator. Considering the scale and light damage in the actual space environment, multiple lasers are designed to meet with the parameters described above and obtain the 486.1-nm blue laser and the $532.2-\mathrm{nm}$ green laser with single pulse energy greater than 1 joule each. A $1^{\circ}-3^{\circ}$ incidence angle 
will be adopted to avoid ocean surface reflection at nadir.

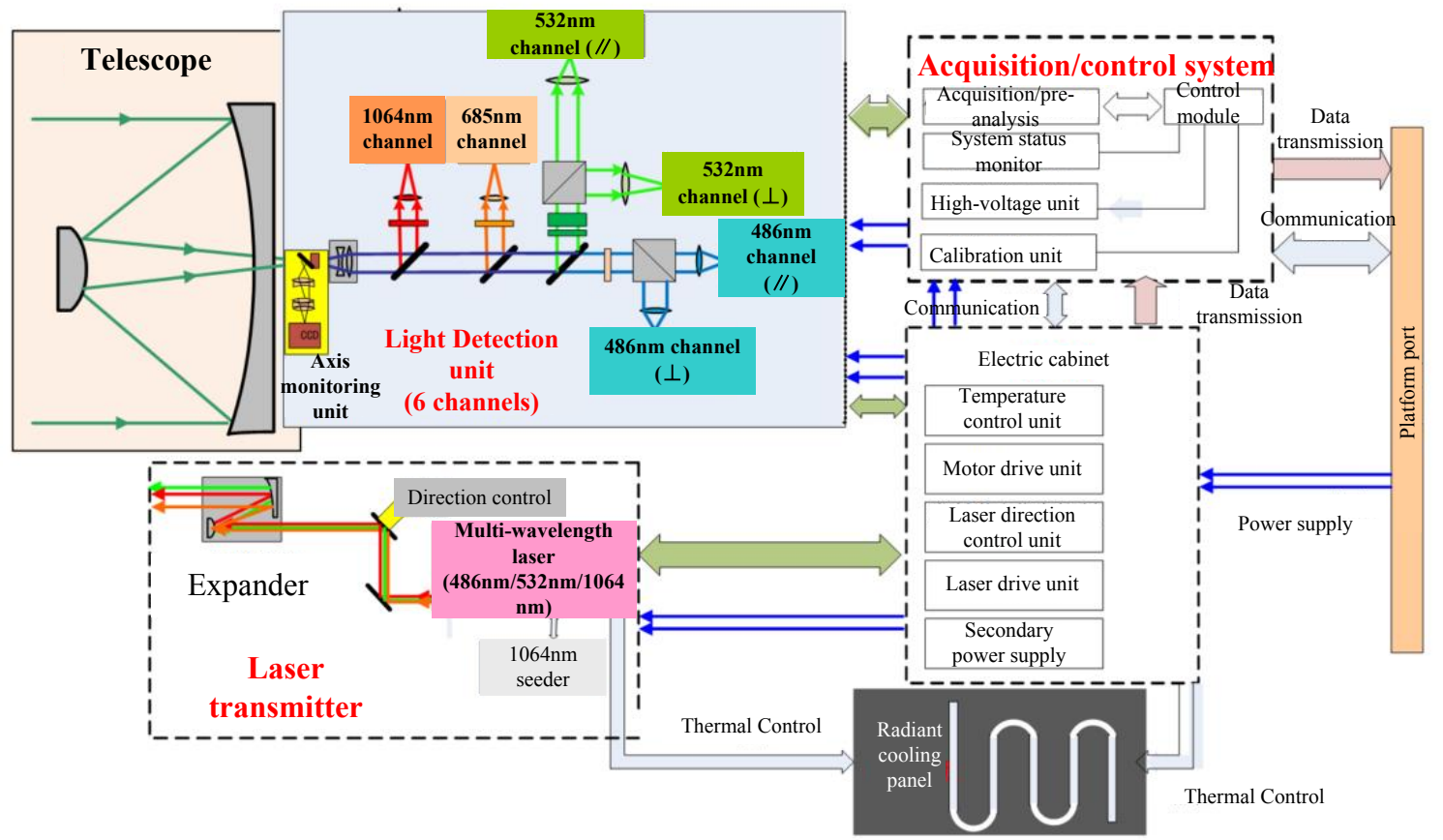

Fig 2. Technology scheme of the spaceborne ocean lidar

The receiving telescope adopts a Cassegrain structure, consisting of a primary mirror and a secondary mirror. The mirror-coated metal reflective film has a reflectivity of more than $95 \%$ in the blue-green band. The telescope has an effective receiving aperture of $1.2 \mathrm{~m}$ and a field of view of $0.2 \mathrm{mrad}$. Under the premise of ensuring the rigidity and stability of the mirror body, the weight of the telescope is to be reduced as much as possible. The primary and secondary mirrors are made of silicon carbide materials with high rigidity, excellent thermal conductivity and high stability. The weight of the optimized telescope will be limited to less than $200 \mathrm{~kg}$.

A single photon counting technique is used to detect laser echoes in multiple channels. With this technology, the echo signal of a single photon can be detected with a count rate of $100 \mathrm{MHz}$. A good signal-to-noise ratio can be obtained by time integration during the detection. In the case of receiving a large number of photons at the same time, the analog signal output can be realized by the accumulation of multi-channel signals. The analog signal is received by the acquisition card with a sampling rate of 1 GSps, and the continuous signal of the underwater vertical profile can be collected. The hybrid method of analog sampling and photon counting will improve detection sensitivity meanwhile provide a high dynamic range.

\subsection{Calibration and validation activities}

Synchronous calibration/validation of the OL sensors onboard the Guanlan satellite is a challenging task. The spaceborne ocean lidar will be mainly calibrated by an airborne lidar. Based on satellite orbit and laser emission direction, the trajectory of laser footprint at the sea surface can be calculated. The airborne lidar verification experiment is then carried out synchronously with satellite measurement in the trajectory direction. A flight altitude of about $4 \mathrm{~km}$ results in a ground path of greater than $50 \mathrm{~km}$, and the trajectory deviation between the footprints of airborne and spaceborne lidars at the sea surface is expected to be less than $2 \mathrm{~km}$. The time deviation between measurements from the two platforms will be less than 0.5 hour. Aside from aircraft based measurements, the calibration method based on the comparison between ocean surface signal and theoretical results (e.g., Cox-Munk theory) using wind speed or surface cross section derived from the radar is also feasible. 
The validation of the Guanlan OL requires simultaneous measurements of the subsurface ocean by the laser satellite and an integrated optical buoy. To do so, an optical multi-parameter profiling system is developed using an automatic winch, along with a ship-borne lidar. The optical buoy will be deployed at the footprint of the satellite orbit, and continuous profiling observations will be carried out when the satellite overflies it. The maximum depth of the profile will exceed $300 \mathrm{~m}$ (almost twice as much as the maximum penetration depth of an ocean lidar) and the vertical resolution will be less than $1 \mathrm{~m}$. Multiple parameter measurements of temperature, salinity, chlorophyll concentration, backscattering coefficient and so on, will be collected and transmitted to the data center in near real time, where an integrated calibration and validation of the Guanlan OL will be implemented in order to reach the level of precision and accuracy.

\section{UNIQUE CHARACTERISTICS AND POTENTIAL BENEFITS}

As one of the first spaceborne ocean lidars and an oceanic counterpart of CALIPSO, the Guanlan OL will be used as an experimental sensor to test a number of new ideas such as a replacement of the nadir-pointing conventional altimeter, a complementary sensor for measuring the ocean component of the global carbon cycle, a new sensor for demonstrating the feasibility of opticalacoustical conversion in the ocean, and an extended sensor for tracing the paths of terrestrial dust into the ocean as well as its biogeochemical effects.

\section{OUTLOOK AND RECOMMENDATIONS}

The ocean has entered the big data stage at the surface while still remains in the small data stage at the bottom. The coexistence of big and small data in terms of ocean measurement is expected to last for a long time. As such, persistent availability of global ocean optical profile data from the Guanlan OL down to the depth of the maximum penetration appears to be extremely valuable, making unique contribution to the observation of oceanic mixed layer. It will also benefit from combination with concurrent in situ surface drifters and Argo floats to routinely provide a systematic monitoring of the upper ocean between 0-2000 m.
In summary, the key objectives of the Guanlan science mission is to extend the penetration depth of space-borne ocean lidar to the vicinity of thermocline, moving a critical step forward towards an ultimate goal of "transparent ocean" which relies on fine resolution, full spectrum, and multidisciplinary oceanographic sensing and observation. Meanwhile, an integrated physicalbiological measurement of the ocean will be achieved, serving as China's novel contribution to the international ocean remote sensing and space oceanography communities.

\section{ACKNOWLEDGEMENTS}

This research is supported by the Pilot National Laboratory for Marine Science \& Technology (Qingdao), China, under grants No. 2018ASKJ01 and 2015ASTP-OS15.

\section{REFERENCES}

[1] L. Fu, et al., Oceanography 23: 14-25 (2010)

[2] D. Winker, et al., Proceedings of the IEEE 84: 164180 (1996)

[3] D. Winker, et al., Bulletin of the American Meteorological Society 91: 1211-1229 (2010)

[4] C. Hostetler, et al., Annual Review of Marine Science 10: 121-147 (2018)

[5] M, Behrenfeld, et al., Geophysical Research Letter 40: 4355-4360 (2013)

[6] L. Fu, et al., Journal of Geophysical Research 119: 4305-4317 (2014)

[7] M, Behrenfeld, et al., Nature Geoscience 10: 118122 (2017) 